\title{
Video Article \\ Scalable 96-well Plate Based iPSC Culture and Production Using a Robotic Liquid Handling System
}

\author{
Michael K. Conway ${ }^{1}$, Michael J. Gerger ${ }^{1}$, Erin E. Balay ${ }^{1}$, Rachel O'Connell ${ }^{1}$, Seth Hanson ${ }^{2}$, Neil J. Daily ${ }^{1}$, Tetsuro Wakatsuki \\ ${ }^{1}$ InvivoSciences, Inc. \\ ${ }^{2}$ Gilson, Inc.
}

Correspondence to: Tetsuro Wakatsuki at tetsuro@invivosciences.com

URL: https://www.jove.com/video/52755

DOI: doi: $10.3791 / 52755$

Keywords: Developmental Biology, Issue 99, iPSC, high-throughput, robotic, liquid-handling, scalable, stem cell, automated stem cell culture, 96well

\section{Date Published: 5/14/2015}

Citation: Conway, M.K., Gerger, M.J., Balay, E.E., O'Connell, R., Hanson, S., Daily, N.J., Wakatsuki, T. Scalable 96-well Plate Based iPSC Culture and Production Using a Robotic Liquid Handling System. J. Vis. Exp. (99), e52755, doi:10.3791/52755 (2015).

\section{Abstract}

Continued advancement in pluripotent stem cell culture is closing the gap between bench and bedside for using these cells in regenerative medicine, drug discovery and safety testing. In order to produce stem cell derived biopharmaceutics and cells for tissue engineering and transplantation, a cost-effective cell-manufacturing technology is essential. Maintenance of pluripotency and stable performance of cells in downstream applications (e.g., cell differentiation) over time is paramount to large scale cell production. Yet that can be difficult to achieve especially if cells are cultured manually where the operator can introduce significant variability as well as be prohibitively expensive to scaleup. To enable high-throughput, large-scale stem cell production and remove operator influence novel stem cell culture protocols using a benchtop multi-channel liquid handling robot were developed that require minimal technician involvement or experience. With these protocols human induced pluripotent stem cells (iPSCs) were cultured in feeder-free conditions directly from a frozen stock and maintained in 96-well plates. Depending on cell line and desired scale-up rate, the operator can easily determine when to passage based on a series of images showing the optimal colony densities for splitting. Then the necessary reagents are prepared to perform a colony split to new plates without a centrifugation step. After 20 passages ( 3 months), two iPSC lines maintained stable karyotypes, expressed stem cell markers, and differentiated into cardiomyocytes with high efficiency. The system can perform subsequent high-throughput screening of new differentiation protocols or genetic manipulation designed for 96 -well plates. This technology will reduce the labor and technical burden to produce large numbers of identical stem cells for a myriad of applications.

\section{Video Link}

The video component of this article can be found at https://www.jove.com/video/52755/

\section{Introduction}

The use of human induced pluripotent stem cells (iPSCs) has increased significantly since their derivation in $2007^{1}$ for compound testing, regenerative medicine and disease modeling ${ }^{2-6}$. This demand comes from iPSC's capacity to yield large numbers of pluripotent cells that can be differentiated into somatic cells at an unparalleled quantity. As directed differentiation techniques improve ${ }^{7-10}$ and development of human cell or tissue modeling and cell therapy increase ${ }^{11-14}$, so does the requirement for mass-production of high quality iPSCs. It is widely cited that among other maladies, myocardial infarction or b-cell functional replacement will require hundreds of millions to billions of iPSC derived somatic cells $^{15-18}$. Furthermore, increasingly complex 3D tissue modeling for drug discovery and therapy will demand large numbers of cells ${ }^{9,13,19}$. In all of these examples, defined, uniform and reproducible iPSCs must be available and straightforward to produce.

In order to produce stem cell derived biopharmaceutics and cells for tissue engineering and transplantation, a cost-effective cell-manufacturing technology is essential. Scaled production of iPSCs has focused on suspension culture ${ }^{20-25}$ or suspension with the use of microcarrier substrates ${ }^{26-28}$ partly because these techniques are successfully deployed for large scale, non-iPSC, eukaryotic based production. Several groups have demonstrated suspension culture systems that yield pluripotent stem cells ${ }^{20,21,23,24,29}$. However, these approaches utilize expensive and complex systems not readily available to researchers developing novel differentiation programs, driving tissue engineering and doing laboratory scale research. Furthermore, suspension and microcarrier iPSC cultures require adaptation and techniques or chemicals not found in traditional adherent iPSC culture such as continuous use of Rho-kinase inhibitor, antifoaming agents and filtration to regulate aggregation. Physical stress to the cells is more prevalent in suspension culture, introduced by mechanical agitation as well as during microcarrier collision. These issues limit the predictability and speed at which newly generated stem cell lines can be cultured in suspension. Others have developed robotic plate handling systems that mimic plate culture techniques ${ }^{30,31}$ but these platforms demand significant investments for equipment and expertise to operate in addition to the fundamental challenges associated with stem cell culture.

The following work describes the development and practice of a scalable iPSC culture system that utilizes a self-contained, standard 8-channel robotic liquid handler and 96-well plates. This method was designed to bridge laboratory scale iPSC culture and high-volume iPSC production (e.g., $10^{7}-1.5 \times 10^{9}$ cells per week per technician) enabling those developing new iPSC technologies to easily scale up production without large 
hardware or labor investments. This method is relatively inexpensive to set up, requires little to no stem cell culture, programming or engineering experience, has a small equipment footprint without the need for a dedicated cell culture hood, and utilizes standard cell culture equipment to enable medium to high throughput automated cell production. The aim was to develop a system capable of scalable stem cell culture that can be utilized by laboratories new to stem cell culture, those who find manual cultivation a barrier to developing their ideas or those wanting an economical means to produce large numbers of stem cells. The platform presented here removes technician influence on stem cell culture and normalizes the feeding and passage procedures to enable consistent stem cell production.

\section{Protocol}

The robotic liquid handling system, which for the following protocols was Gilson's pipetmaX, facilitates automated, scalable stem cell culture and production while maintaining traditional adherent culture conditions in the 96-well plate format. Like traditional 6-well plate culture, iPSCs are grown with this protocol as a monolayer of distinct colonies but in the 96-well plate format. Each well can be isogenic or distinct and either configuration can be cultured in parallel since the robot can keep each well segregated. The typical robotic iPSC culture routine has two phases: a feeding program where cultures are fed on a customizable schedule and a passage program where the user chooses the dissociation method and splitting ratio thereby controlling seeding density and scale rate. The following protocols describe how a new culture is started, how feeding and passage are accomplished and the supplementary programs that facilitate iPSC culture.

Note: Please see the Materials List for specific recommendations on the matrix coating material and dissociation reagents validated for the following protocols.

\section{Preparing Extracellular Matrix Gel Coated 96-well Plates}

1. Remove the packaging from six new, RT 96 -well plates and place them on the liquid handling robot bed in positions $2,3,5,7,8,9$ (see Figure 1A for bed positions).

2. Place a pre-chilled, $4{ }^{\circ} \mathrm{C} 4$-well trough in bed position 6

3. Load column 12 of the tip rack in bed position 1 with pre-chilled, $4^{\circ} \mathrm{C} 200 \mu$ pipet tips.

4. Fill the first (left most) trough well in bed position 6 with $25 \mathrm{ml} 4{ }^{\circ} \mathrm{C}$ DMEM/F12 by pouring an aliquot using sterile technique. Alternatively, use a pipet to complete the transfer.

5. Remove the 96-well plate lids and slide each into the specially designed plate lid holding rack (PLHR, see Figure 1A). Avoid contact with the inside of plate lids.

Note: Handling the inside of a plate lid or stacking the plate lids where the inside of one lid touches the outside of another is an excellent route for contamination.

6. Using the robotic control touch pad, press the following series of buttons to start the well pre-wetting process:

1. Tap "Run a Protocol".

2. Select "extracellular matrix gel Prewet" and tap next.

3. User decision: Tap "test run" to use the step-by-step wizard to pre-test the selected program.

Note: The robot does not run but rather the software tests the protocol for software problems. With established protocols, tapping, "skip setup" is acceptable.

4. Tap "Run Protocol".

7. During the pre-wetting process (step 1.6) continue to step 1.8 .

8. Thaw on ice one $4 \mathrm{mg}$ aliquot of growth factor reduced extracellular matrix gel (GFRM) contained in a $50 \mathrm{ml}$ conical tube stored at $-80{ }^{\circ} \mathrm{C}$. Keep the aliquot on ice until ready to use.

Note: The GFRM will solidify if allowed to come to RT and will not be useful.

9. Resuspend the $4 \mathrm{mg}$ GFRM aliquot in $24 \mathrm{ml} 4{ }^{\circ} \mathrm{C}$ DMEM/F12 with a $25 \mathrm{ml}$ glass pipet. When transferring the DMEM/F12, pause for 2-3 sec to allow the pipet to cool prior to resuspending the GFRM aliquot.

10. When the pre-wetting process (step 1.6) is complete, continue to step 1.11 .

11. Transfer the $24 \mathrm{ml}$ of DMEM/F12 GFRM mixture to the fourth well of the trough in bed position 6 .

12. Using the robotic control touch pad, press the following series of buttons to start the extracellular matrix gel coating process:

1. Tap "Run a Protocol".

2. Select "extracellular matrix gel aliquot" and tap next.

3. User decision: Tap "test run" to use the step-by-step wizard to pre-test the selected program.

Note: The robot does not run but rather the software tests the protocol for software problems. With established protocols, tapping, "skip setup" is acceptable.

4. Tap "Run Protocol".

13. When step 1.12 is complete, replace the plate lids.

14. Transfer the extracellular matrix gel coated 96 -well plates to a $37^{\circ} \mathrm{C}, 5 \% \mathrm{CO}_{2}$, humidified incubator and hold there for $24 \mathrm{hr}$ at which point the plates will be ready to use or stored.

Note: Under extenuating circumstances, the newly coated extracellular matrix gel plates may be used after $15-30$ min of incubation but $\mathrm{O} / \mathrm{N}$ to $24 \mathrm{hr}$ incubation is recommended.

15. Repeat protocol steps 1.1, 1.3, 1.5-1.6, 1.10, and 1.12-14 until all of the DMEM/F12 with GFRM is used.

\section{Storing Extracellular Matrix Gel Coated 96-well Plates}

1. Remove extracellular matrix gel coated 96 -well plates from the $37{ }^{\circ} \mathrm{C}, 5 \% \mathrm{CO}_{2}$, humidified incubator.

2. Optional: Allow the extracellular matrix gel coated 96 -well plates to come to RT before proceeding to step 2.3 . Note: This optional step will reduce condensation build up when the plates are placed at $4{ }^{\circ} \mathrm{C}$. 
3. Place the plates on the liquid handling robot bed in positions $2,3,5,7,8,9$ (see Figure 1A for bed positions).

4. Load column 12 of the tip rack in bed position 1 with RT $200 \mu \mathrm{l}$ pipet tips.

5. Place a four trough reservoir in bed position 6 .

6. Fill well 4 (rightmost) of the reservoir with RT DMEM/F12.

7. Remove the 96-well plate lids and slide each into the PLHR.

8. Using the robotic control touch pad, press the following combination of buttons:

1. Tap "Run a Protocol".

2. Select "extracellular matrix gel aliquot" and tap next.

3. User decision: Tap "test run" to use the step-by-step wizard to pre-test the selected program.

Note: The robot does not run but rather the software tests the protocol for software problems. With established protocols, tapping, "skip setup" is acceptable.

4. Tap "Run Protocol".

9. Once complete, replace the plate lids and remove the plates from the machine bed.

10. Wrap around the entire side of each extracellular matrix gel coated 96-well plate (where the lid meets the plate) with a one inch by 6 inch paraffin film strip. Be sure to stretch the paraffin film to create an airtight seal.

11. Optional: Label the plates with the date of completed extracellular matrix gel coating, the extracellular matrix gel lot number, user name and any other information.

12. Store the plates at $4{ }^{\circ} \mathrm{C}$ where they will be good to use for up to two weeks.

Note: Plates have been successfully used beyond the two week limit, however, it is not recommended.

\section{Performing a Stem Cell Colony Seeding Density Gradient in the 96-well Plate Format from a Live or Frozen Culture: How to Start or Restore Normal Passage Intervals to a Stem Cell Line}

1. If starting with a frozen culture, proceed to step 3.2. If starting with a live stem cell culture already on a plate, begin at step 3.5.

2. Thaw the frozen culture by swirling the tube in a $37^{\circ} \mathrm{C}$ water bath until only a small chunk of ice remains.

3. Optional: Dilute the thawed cells into $10 \mathrm{ml} \mathrm{RT} \mathrm{stem} \mathrm{cell} \mathrm{growth} \mathrm{media} \mathrm{(SCGM),} \mathrm{centrifuge} \mathrm{for} 2$ min at $300 \times \mathrm{g}$, aspirate the supernatant media and resuspend the stem cell pellet in $7 \mathrm{ml}$ fresh SCGM containing $10 \mu \mathrm{M}$ Y27632.

Note: This step eliminates carryover of the freezing media.

4. Proceed to Step 3.6.

5. If starting with live, already plated stem cells: passage the cells as normal using enzymatic or non-enzymatic dissociation. Try to harvest a total of 1.5-3 million cells; usually one well of a six-well plate. Centrifuge the harvested cells for 2 min at $300 \times \mathrm{g}$, aspirate the supernatant media and resuspend the stem cell pellet in $7 \mathrm{ml}$ fresh SCGM containing $10 \mu \mathrm{M}$ Y27632. Proceed to Step 3.6.

6. Ensure the cells, either from a frozen or live culture, are suspended in $7 \mathrm{ml} \mathrm{RT} \mathrm{SCGM} \mathrm{with} 10 \mu \mathrm{M}$ Y27632.

7. Load column 12 of the tip rack in bed position one with RT $200 \mu$ l pipet tips.

8. Place a four trough reservoir in bed position 2 .

9. Place one new extracellular matrix gel coated 96 -well plate pre-warmed to $37^{\circ} \mathrm{C}$ in bed position 5 and remove the lid placing it in the PLHR.

10. Transfer the $7 \mathrm{ml}$ of resuspended cells to well 3 of the reservoir by pipet or sterile pour.

1. Using the robotic control touch pad, press the following combination of buttons:

2. Tap "Run a Protocol".

3. Select "Seeding Density Gradient" and tap next.

4. User decision: Tap "test run" to use the step-by-step wizard to pre-test the selected program.

Note: The robot does not run but rather the software tests the protocol for software problems. With established protocols, tapping, "skip setup" is acceptable.

11. Tap "Run Protocol".

12. When the dilution series is complete, re-cover the plate and place in a $37^{\circ} \mathrm{C}, 5 \% \mathrm{CO}_{2}$, humidified incubator until the next feeding

13. Optional: Label the plate with the date, strain information, user name, media type and other pertinent information.

14. Over the next several days, feed the 96-well plate as required using Protocol 4 below.

Note: A typical stem cell plate will require a full media change once every $24 \mathrm{hr}$ and the SCGM does not contain Y27632 for feeding; only during the initial seeding after passage. Prior to feeding, check the plate for contamination and colony density. See step 3.16 for more on monitoring colony density.

15. Over the next few days, several columns near the center of the plate (usually columns 5-8) will approach an ideal density for passage; to identify this density, compare the 96-well plate to the density range shown in Figure 3B with the following information in mind:

1. Passage when the space between the majority of the colonies is approximately $25 \%$ of adjacent colony diameter.

Note: The rationale to identify the time to passage is that another cycle of feeding and growth would result in colonies making contact, which should be avoided.

16. To start a uniformly diluted plate and begin regular culture, select a column that is at the ideal density and passage. See Protocol 5 to passage.

\section{Feeding 96-well Plate Stem Cell Colonies}

Note: The following protocol describes the feeding of one 96 -well stem cell colony plate. The technique can be scaled up to accommodate 6 total plates in parallel.

1. Remove the 96 -well stem cell colony plate from the $37^{\circ} \mathrm{C}, 5 \% \mathrm{CO}_{2}$, humidified incubator. 
2. Check the plate for contamination and cell density. In order to feed, ensure that the colony density is below the ideal passage density. See Figure 3B for information on density.

Note: Contamination may present as turbid media and be accompanied by an unpleasant smell. Small, obviously non-iPSC aggregates may also be visible under microscopic inspection. To evaluate cell density, see step 3.16 .1 above.

3. Place the 96-well stem cell colony plate in bed position 3 on a sloped, $37^{\circ} \mathrm{C}$ ramp.

4. Load column 12 of the tip rack in bed position 1 with RT $200 \mu$ l pipet tips.

5. Place a 4-well trough in bed position 2 .

6. Fill well 3 of the trough in bed position 2 with $8 \mathrm{ml}$ fresh, RT SCGM without Y27632 either by sterile pour or pipet transfer.

7. Remove the 96-well stem cell colony plate lid placing it in the PLHR.

8. Using the robotic control touch pad, press the following combination of buttons:

1. Tap "Run a Protocol".

2. Select "Colony Feed One Plate" and tap next.

3. User decision: tap "test run" to use the step-by-step wizard to pre-test the selected program.

Note, the robot does not run but rather the software tests the protocol for software problems. With established protocols, tapping, "skip setup" is acceptable.

4. Tap "Run Protocol".

9. When the feeding protocol is complete, re-cover the 96 -well stem cell colony plate and return to the $37{ }^{\circ} \mathrm{C}, 5 \% \mathrm{CO}_{2}$, humidified incubator until the next feeding or passage is required. This is usually required after $24 \mathrm{hr}$.

Note: It is recommended to record the feeding event on the plate cover with the date, media type, user name and other pertinent information.

\section{Passaging 96-well Plate Stem Cell Colonies}

1. After several feeding cycles the 96-well stem cell colony plate will be ready to passage. This protocol describes passage of one column to one 96-well plate, representing a 1:12 split ratio. The user can define the split ratio and select any number of wells or columns to split, including choosing wells that may not be adjacent.

2. Compare the 96-well stem cell colony plate density to Figure 3B to determine whether to passage. The ideal passage density is when the space between the majority of colonies is approximately $25 \%$ of adjacent colony diameter or a subsequent feeding would result in the colonies growing into one another.

3. Examine the 96-well stem cell colony plate under a microscope to ensure there is no contamination.

4. Place the 96 -well stem cell colony plate in bed position 3 on a sloped, $37^{\circ} \mathrm{C}$ ramp.

5. Load columns $8-12$ in the tip loading rack in bed position 1 with RT $200 \mu$ pipet tips.

6. Place a 4-well trough in bed position 2 .

7. Leave trough well 1 empty, put $8.5 \mathrm{ml} \mathrm{SCGM}+10 \mu \mathrm{M}$ Y27632 in well 2, put $3.5 \mathrm{ml} 30 \%$ proteolytic and collagenolytic dissociation reagent (or EDTA based dissociation reagent) in well 3 , put $4.5 \mathrm{ml}$ PBS in well 4.

Note: All reagents can be at RT or $37^{\circ} \mathrm{C}$. RT proteolytic and collagenolytic dissociation reagent at $30 \%$ diluted with PBS was used for the Adipose and Fibroblast derived iPSC culture described here.

8. Place one new, pre-warmed $37^{\circ} \mathrm{C}$ extracellular matrix gel coated 96-well plate in bed position 5 .

9. Remove the 96-well stem cell colony plate lid, as well as the new 96-well plate lid and place them both in the PLHR.

10. Using the robotic control touch pad, press the following combination of buttons:

1. Tap "Run a Protocol".

2. Select "Colony Split 1:12 Column 1" and tap next.

3. User decision: Tap "test run" to use the step-by-step wizard to pre-test the selected program.

Note: The robot does not run but rather the software tests the protocol for software problems. With established protocols, tapping, "skip setup" is acceptable.

4. Tap "Run Protocol”.

11. Once the passage protocol has finished, re-cover both plates.

12. Optional: Label the new plate with the requisite information (i.e., date, cell line, passage number, media type, user name etc.).

13. Return both plates to the $37^{\circ} \mathrm{C}, 5 \% \mathrm{CO}_{2}$, humidified incubator until the next feeding or passage is required. Note: The next feeding is typically after $24 \mathrm{hr}$.

Note: Cells and colonies should attach to the plate within 2-3 hr of splitting and look very spread out. This is due to the presence of Rhokinase inhibitor and the cells will condense and exhibit the characteristic stem cell morphology (i.e., cobblestone packed colonies with small cell volume to big nucleus ratio) after the first Rho-kinase inhibitor free feeding.

\section{Harvesting 96-well Plate Stem Cells for Production}

Note: Stem cell colonies may be harvested for use at any point during culture. Usually this occurs when the cells are ready to passage (see protocol 5). This protocol describes how to harvest eleven columns from a 96-well stem cell colony plate.

1. Examine the 96-well stem cell colony plate under a microscope to ensure there is no contamination.

2. Place the 96 -well stem cell colony plate in bed position 3 on a sloped, $37^{\circ} \mathrm{C}$ ramp.

3. Load columns 11 and 12 in the tip loading rack in bed position 1 with RT $200 \mu$ pipet tips.

4. Place a 4-well trough in bed position 2 .

5. Leave trough well 1 empty, put $8.5 \mathrm{ml} \mathrm{SCGM}$ in well 2, put $3.5 \mathrm{ml} 30 \%$ proteolytic and collagenolytic dissociation reagent (or EDTA based dissociation reagent) in well 3 , put $4.5 \mathrm{ml}$ PBS in well 4 .

Note: All reagents can be at RT or $37^{\circ} \mathrm{C}$.

6. Remove the 96-well stem cell colony plate lid and place in the PLHR.

7. Using the robotic control touch pad, press the following combination of buttons: 
1. Tap "Run a Protocol".

2. Select "Colony Harvest Columns 2-12" and tap next.

3. User decision: Tap "test run" to use the step-by-step wizard to pre-test the selected program.

Note: The robot does not run but rather the software tests the protocol for software problems. With established protocols, tapping, "skip setup" is acceptable.

4. Tap "Run Protocol".

Note: Once the collection procedure is complete, the cells will be in trough well 2 and ready for collection.

Representative Results

Development of a plate based robotic stem cell culture platform.

The demand for iPSCs is growing due to their utility in drug development and regenerative medicine. Yet scalable production has focused on suspension culture ${ }^{32}$ in relatively complex equipment that excludes many researchers and diverges from established adherent culture methods. Rather than drastically alter proven plate based stem cell culture methods, such as switching to a suspension culture or using a microcarrier, we focused on miniaturizing and automating our existing adherent iPSC culture techniques. The first aim was to automate feeding and passaging to normalize the entire culture process. A platform capable of rapid scale up with existing technology was also required. To achieve these goals a method was devised to culture stem cells in a 96-well plate utilizing an automated liquid handling system (Figure 1). The protocols developed here with the liquid handling robot for feeding and passage do not require a centrifugation step or continuous monitoring by a technician. These protocols were developed with two feeder free iPSC lines; one derived from fibroblasts and the other from adipose cells ${ }^{33}$.

The computer controlled liquid handling system (Figure 1A) consists of a flatbed that moves front and back. The bed has nine, approximately 5 x 3.3 inch numbered recesses with pressure retaining clips to accept standard cell culture plates, liquid reservoirs and other custom hardware. The pipet head moves left to right (perpendicular to bed movement) as well as up and down. Together with the bed, the pipette head can be programmed to remove or deliver media anywhere on the bed after picking up pipette tips from a refillable rack. If necessary, each of the 96wells can be kept separate to eliminate cross contamination. In the present configuration, 0 to $200 \mu$ of media can be moved by each tip of the pipet head, but other volume ranges are possible based on tip size.

When passaging stem cells in standard plates, enzymatic or chemical dissociation typically requires incubation at $37^{\circ} \mathrm{C}$. Initial testing confirmed dissociation with a proteolytic and collagenolytic dissociation reagent or an EDTA based reagent performed better at $37^{\circ} \mathrm{C}$ than RT both for time to dissociation and homogeneity of colony size produced for our two 96-well iPSC lines (data not shown). To automate the split process and avoid moving plates in and out of an incubator, sloped, temperature controlled ramps that accept and reproducibly position standard culture plates were built (Figure 1A). When the ramps were heated to $37^{\circ} \mathrm{C}$, a proteolytic and collagenolytic dissociation reagent or EDTA based dissociation of the iPSCs from 96-well plates was comparable to plates placed in a $37^{\circ} \mathrm{C}$ incubator (data not shown). The sloped ramps were also utilized to enable pipet tips to collect an entire well's contents (less than $5 \mathrm{uL}$ residual volume) by reaching the lowest point in the well whereas aspiration from a flat plate left a residual volume of approximately 10-15 $\mu$, resulting in cell loss. The sloped ramp also allowed the wells to be washed (triturated) by ejecting media at the top of the sloped well and to collect it at the bottom.

\section{Robotic culture of stem cells in the 96 -well plate format; feeding and passaging.}

Culture of iPSCs using the robotic liquid handling system has two phases; passage and feeding. When a colony is ready to passage, it is split at a predetermined ratio to seed a new plate which is then fed at regular intervals until it is ready to passage again (Figure 1B). Frozen or non-96well cultures can be started in the 96-well format and immediately enter the passage/feed cycle. Cells that are not used to maintain the colony, which is the majority of a plate, are harvested for other uses and represent the production component of this system.

Feeding 96-well cultured iPSCs is accomplished when some or all of the media is removed after a period of culture and deposited in a waste reservoir. Then fresh media is aliquoted to the same plate. The robot can use new tips and media troughs to segregate every well for fully customizable feeding, including blending conditioned media with new media.

Typical passage of stem cells requires a method of dissociation and often a centrifugation step. The protocols described here aims to normalize dissociation and eliminate centrifugation. The passage protocol begins when the robot delivers dissociation reagent to 96-well plates mounted on $37^{\circ} \mathrm{C}$ sloped ramps. After a preset time, the dissociated cells are collected with a washing action where the user has control over the position, repetition, volume, and trituration rate. Enzyme time, trituration rate and the number of trituration repetitions were sufficient to vary dissociated colony size and homogeneity (Figure 2), but each parameter is adjustable to fit a given cell line's requirements. This control removes variability introduced by technicians who pipet with varying rates, positions, and repetitions. Once the dissociated cells are collected and pooled in a reservoir with fresh media, they are distributed to a new plate. To avoid centrifugation and seeding problems from substrate degradation or cell death due to enzyme action, dissociation reagent was diluted to the minimum point of acceptable dissociation that resulted in reliable seeding. This technique was effective for a proteolytic and collagenolytic dissociation reagent in mTeSR 1 medium $^{34}$, which has a relatively high protein content, but also effective in low protein media like Essential- ${ }^{35}$, both of which are compatible with this system (E8 data not shown).

Controlling the seeding density of stem cells after passage is paramount to routine and successful stem cell culture. Seeding too low or high can result in ectopic differentiation and loss of pluripotency in addition to causing irregular passage intervals. Typical stem cell culture relies on ratio splitting where one well of a 6 -well plate is used to seed an entire new 6 -well plate (a 1:6 split). With the liquid handling robot this ratio can be adjusted to suit the needs of the user (e.g., 1:6, 1:9, 1:12, etc.) and it has the most influence on the passage interval. The robot can harvest less than one column, one entire column ( 8 wells), or more than one column depending on the user's needs, which should be determined empirically. The adipose and fibroblast derived iPSCs maintained a regular three day feeding schedule with passage on the fourth day when split 1:12. In this case one column was harvested and used to seed one new 96-well plate, creating a 1:12 split with each cycle (Figure 3A). The remaining 
11 wells were then available for downstream applications. To harvest these 11 production wells, the passage protocol was repeated except the cells were deposited into a trough for collection. Alternatively, the cells can be left on the plate and used directly.

To achieve consistent seeding density passage to passage without counting, and maintain a routine splitting schedule, our protocols call for splitting the same predetermined number of columns when the colony is at an ideal density for passage. To help users identify the proper density for passage, a series of images was generated showing a range of densities with an ideal passage density highlighted (Figure 3B). To determine when to passage, a technician examines their plate and compares it to the density image scale. The ideal density to passage was determined from culture of the adipose and fibroblast derived iPSCs and found to be when the space between most colonies was about $25 \%$ of adjacent colony diameter. It is important that the colonies be homogenously distributed. This amount of colony separation correlates with the maximum desirable density because one additional feeding cycle would result in colonies touching, which is a trigger for ectopic differentiation.

A necessary protocol developed here addresses how to start a culture in the 96-well format and how to reset a culture after a density problem. When a new culture is started, the best seeding density and number of feeding cycles before passaging are unknown. To ensure a usable density after seeding, the robot distributes existing or thawed cells across a 96-well plate in a serial dilution (Figure 4A). Example results of such a gradient are shown in Figure 4B-E. After several feeding cycles, some columns approach the ideal density to split, at which point a technician uses the robot to seed a uniformly diluted plate to start regular culture. This density gradient protocol was also used to restore regular passage intervals and colony homogeneity to an irregular plate. If passage is delayed or missed, colony density and size become too high, whereas if passage is too early, colony density is too low and individual colonies may become too large without being evenly distributed across the well. The density gradient protocol resolves these issues and allows the user to restart by picking an ideally seeded set of wells.

\section{Two iPSC lines remained pluripotent after 3 months of robotic culture.}

Maintenance of stem cell pluripotency can be adversely affected by culture conditions ${ }^{36,37}$. To evaluate whether adipose and fibroblast derived iPSC lines (a-iPSC, f-iPSC, respectively) maintained pluripotency when cultured robotically in 96-well plates for an extended period of time, the two lines were cultured on the liquid handling robot as described above for 3 months and then pluripotency markers were examined. For this period both lines were passaged with a proteolytic and collagenolytic dissociation reagent greater than 20 times without centrifugation. Fixed 96-well plates from a-iPSC and f-iPSC lines stained for Oct4 and Nanog exhibited nuclear accumulation of these markers while Ssea-4 was observed on the cell surface (Figure 5A-L). This is consistent with previous reports for pluripotent iPSCs ${ }^{38-41}$. Counterstaining with DAPI did not reveal any additional cells that were not also positive for the three pluripotency markers. Chromosomal abnormalities are commonly observed during stem cell culture ${ }^{42,43}$ but may not affect the distribution of pluripotency markers like those shown in Figure 5A-L. Karyotype analysis revealed both robotically passaged iPSC lines had 46 normal chromosomes, suggesting the robotic culture method did not introduce chromosomal instability beyond what might normally occur (Figure $5 \mathrm{M}-\mathrm{N}$ ).

To probe established stem cell gene expression markers ${ }^{1,41,44,45}$ in the robotically cultured iPSC lines, total RNA was collected in parallel to staining and karyotype analysis. Both 96-well plate iPSC lines had similar expression of pluripotency markers NANOG, POU5F1 and REX1 whereas cardiomyocytes differentiated from each line did not, nor did a separate line of human dermal fibroblasts (HDFs) (Figure 6). The cardiomyocytes derived from both lines were MYL7 positive whereas the HDFs and stem cells did not express MYL7. Both iPSC lines were differentiated into cardiomyocytes with the small molecule, Wnt pathway manipulation technique described recently ${ }^{46}$. When stained, cardiomyocytes from both lines were cTnT positive with clear sarcomere formation (Figure 7). Differentiation into cardiomyocytes in the 96-well format was successful in greater than $80 \%$ of the wells (data not shown). Together, these data suggest 3 months and more than 20 passages of robotic culture resulted in chromosomally normal cells exhibiting a transcription program consistent with pluripotency that were also capable of differentiation into cardiomyocytes.
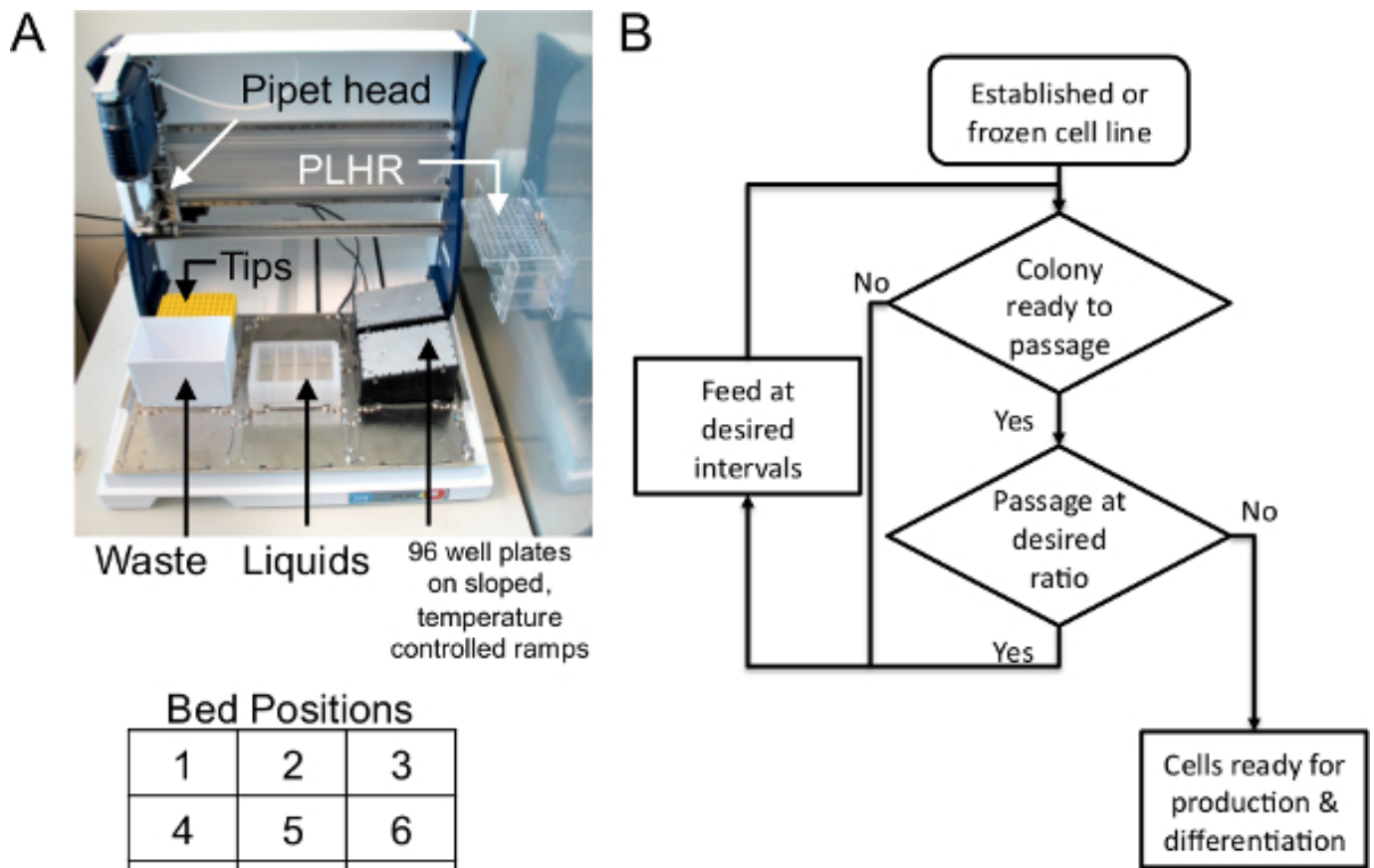

Bed Positions
\begin{tabular}{|c|c|c|}
\hline 1 & 2 & 3 \\
\hline 4 & 5 & 6 \\
\hline 7 & 8 & 9 \\
\hline
\end{tabular}

Cells ready for differentiation 
Figure 1. Overview of robotic stem cell culture equipment and typical iPSC culture routine. (A) Robotic liquid handling system shown with typical bed layout for 96 -well stem cell culture. New sterile pipet tips (Tips), removable tip waste container (Waste), multi-well autoclavable trough to hold required liquid reagents (Liquids), 96-well plates are positioned and supported on a temperature controlled, sloped ramp (96-well plates), 8 channel robotic pipet head that moves left/right and up/down (Pipet head). Plate lid holding rack (PLHR). Bed position numbers shown below in table. (B) Robotic iPSC culture has two phases: Feeding and Passage. The cycle of cell production starts when a colony plate is ready to passage. The user chooses the desired ratio to passage at and the robot seeds a new group of 96-well plates then fed at regular intervals until ready for passage again. When the plates are ready to passage, most of each plate is not used to seed subsequent colony plates and is available for other uses, thus representing the production phase of the system. Frozen or existing cell lines can be introduced at any time and maintained in the feed/passage cycle. Please click here to view a larger version of this figure.
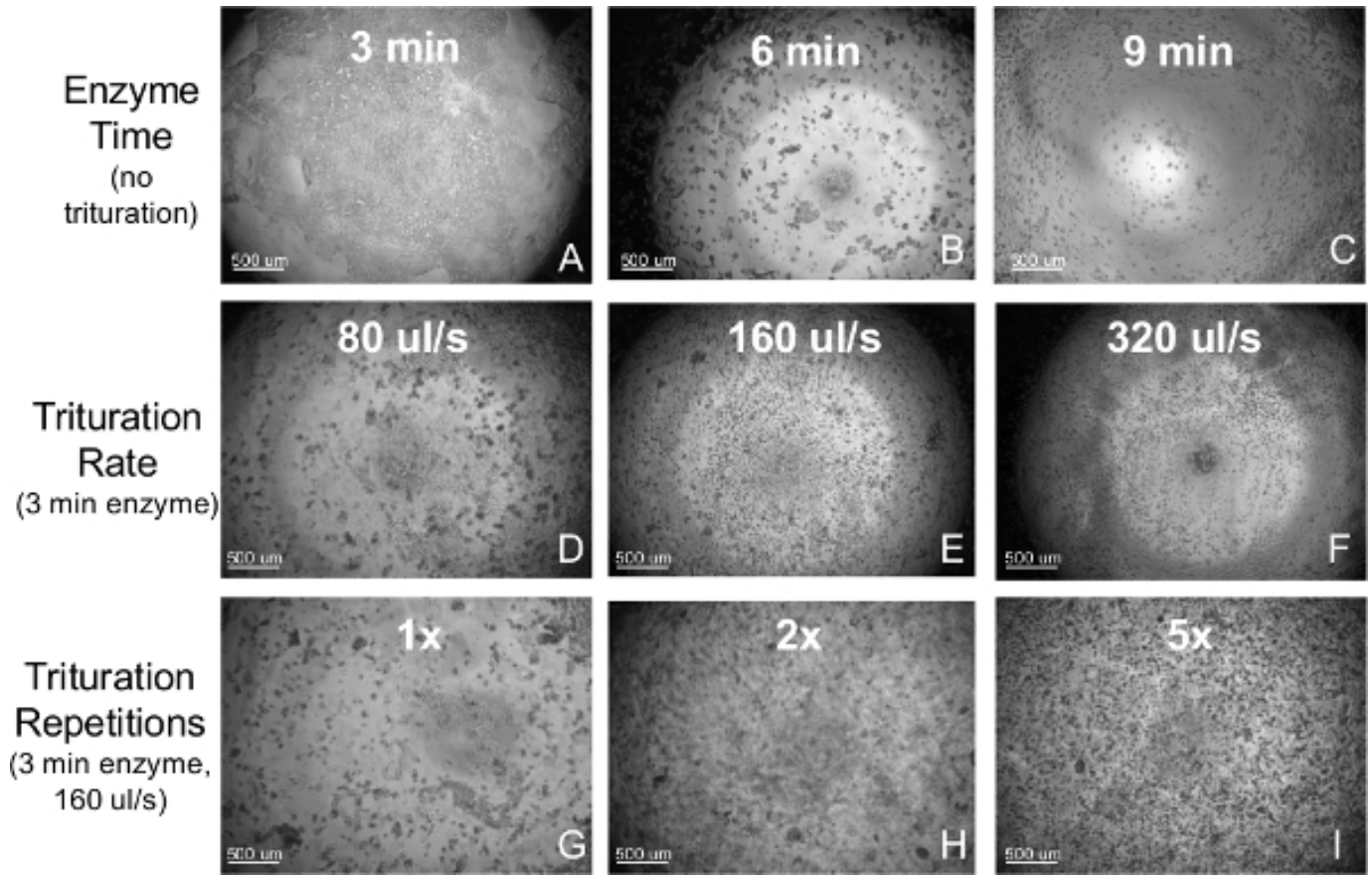

Figure 2. Robotic liquid handling parameters can be used to control iPSC colony dissociation characteristics. Each representative image shows fibroblast derived iPSCs dissociated after the indicated treatment while still suspended in the 96-well. (Top Row, A-C) Enzyme Time, no trituration. Fibroblast derived iPSCs grown in 96-well plates were subjected to increasing times of proteolytic and collagenolytic dissociation reagent dissociation and no robotic trituration was performed. (Middle Row, D-F) Trituration rate, 3 minutes enzyme. Cells were exposed to three minutes of proteolytic and collagenolytic dissociation reagent treatment and then $175 \mu \mathrm{l}$ growth media was applied and each well pipetted up and down once at the indicated rate. $\mu \mathrm{l} / \mathrm{sec}=$ microliters per second. (Bottom Row, G-I) Trituration Repetitions, 3 minutes enzyme, $160 \mu \mathrm{l} / \mathrm{sec}$. Cells were exposed to proteolytic and collagenolytic dissociation reagent for 3 minutes, $175 \mu$ growth media was applied, then triturated at $160 \mu \mathrm{l} / \mathrm{sec}$ for the indicated number of repetitions. Please click here to view a larger version of this figure. 
A

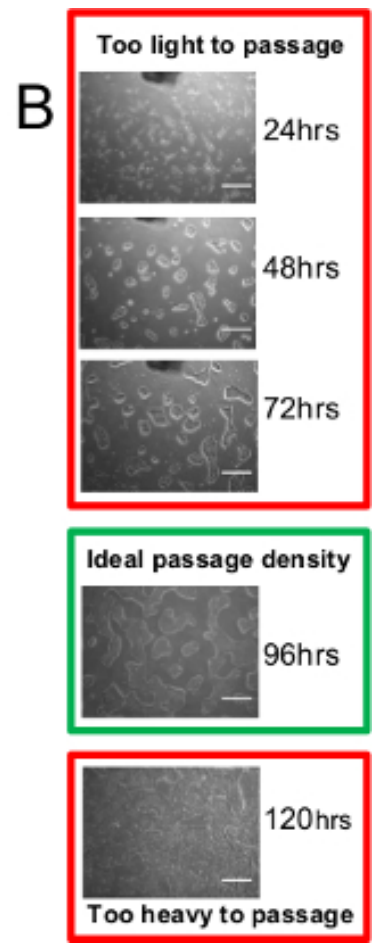

Figure 3. Typical adipose and fibroblast iPSC colony maintenance scheme and a series of colony density images to determine when to passage to maintain a regular feeding/passage cycle. (A) Beginning with an adipose or fibroblast 96-well iPSC colony ready to passage, one column of cells was passaged on the robot without centrifugation and diluted to 12 new columns which were fed at specific intervals thereafter until the plate was ready to passage again. For cell production, columns not used to maintain the colony were passaged and collected for subsequent use. Any number of columns may be passaged to control expansion rate. (B) To determine when to passage, a series of density images captured every $24 \mathrm{hr}$ was provided to users. In the top red box (within $72 \mathrm{hr}$ after initial seeding) the colony is not dense enough to passage and should be fed. When the density matches that shown in the green box (at $\sim 96 \mathrm{hr}$ ), the colony is at an ideal density for passage. By $120 \mathrm{hr}$, the colony is too dense to passage and this scenario should be avoided. The latter may be resolved with a seeding density gradient but routine culture at this density is strongly discouraged. White bar equals $200 \mu \mathrm{M}$. Please click here to view a larger version of this figure. 

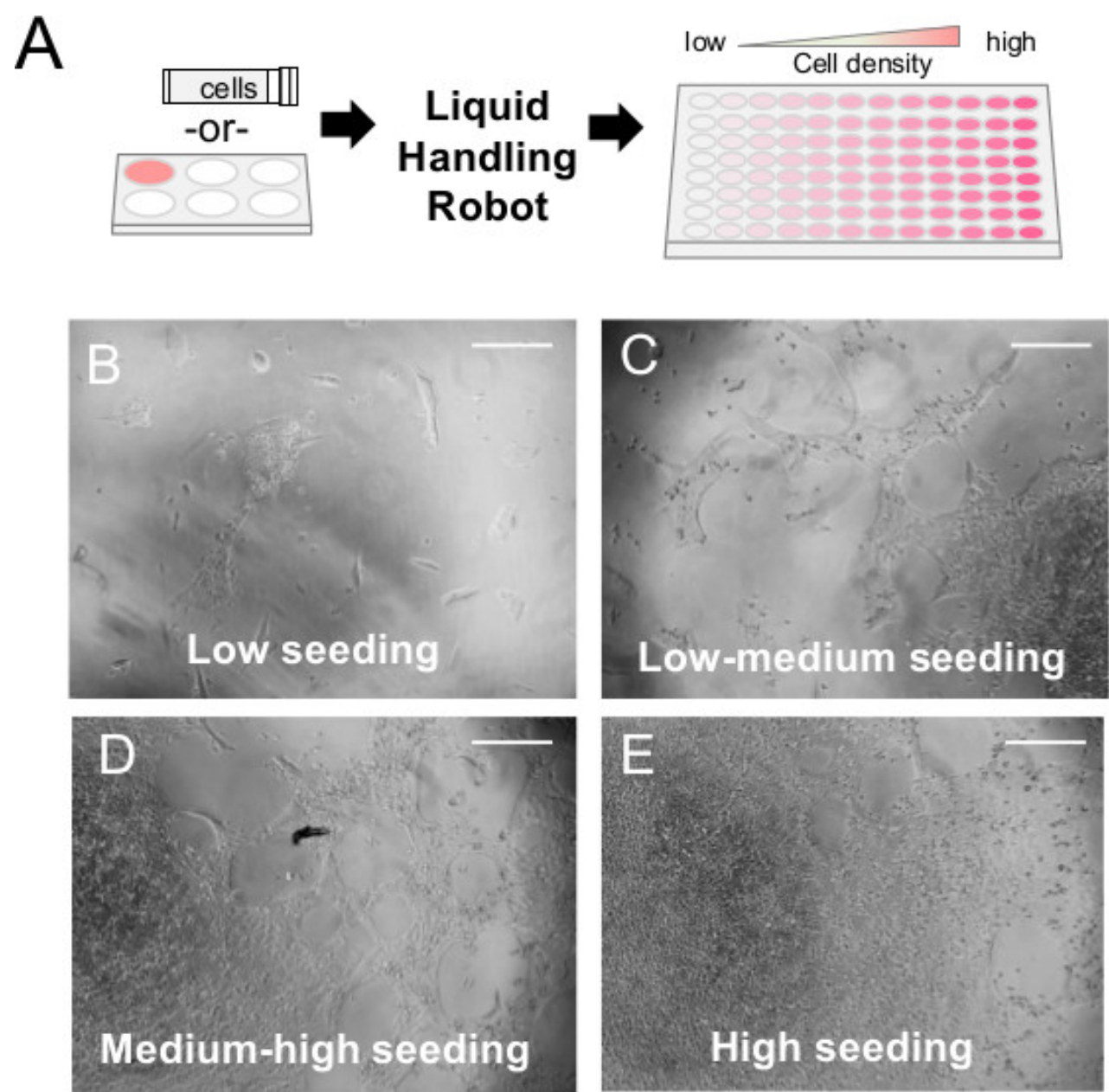

Figure 4. 96-well robotic culture system allows users to start cultures from frozen or active cell lines and perform a seeding gradient. (A) A cell solution is prepared by the user from a frozen cell stock or after harvesting an active culture and placed on the robot. The robotic system then performs a serial dilution protocol to distribute the initial cell solution along the 96-well plate with the density gradient distributed by column. (B-E) Examples of cell density from four of the twelve columns from A, $48 \mathrm{hr}$ after the density gradient seeding protocol. White lines equal $225 \mu \mathrm{m}$. Please click here to view a larger version of this figure. 
96 well adipose iPSC
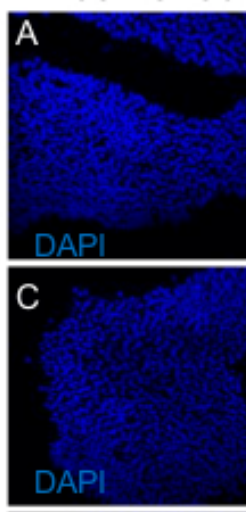

E

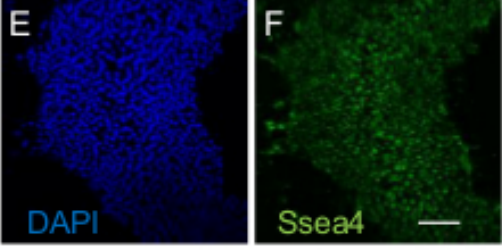

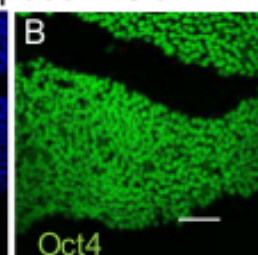

D
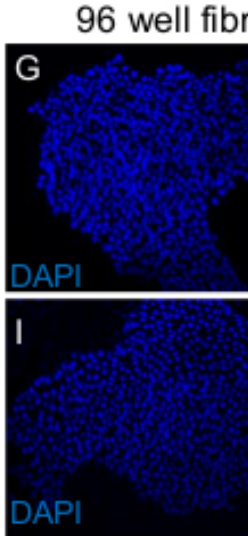

Oct4
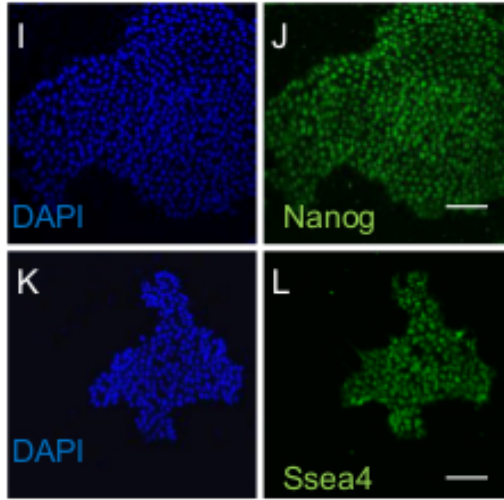

M

\section{$\mathrm{N}$}

96 well adipose iPSC p22 46XX

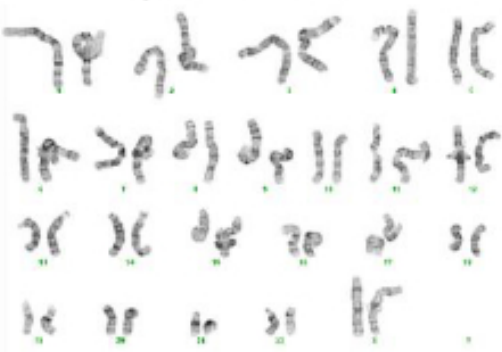

96 well fibroblast iPSC p23 46XY
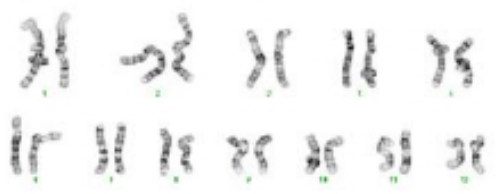

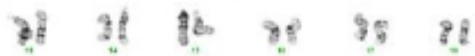

$36 \quad 38 \quad 30 \quad 96 \quad$ है ?

Figure 5. Pluripotency is maintained for adipose and fibroblast derived iPSC lines during 96-well robotic culture for 22 (adipose) or 23 (fibroblast) passages ( $\sim 3$ months). (A-L) Adipose and fibroblast derived iPSC cell lines were robotically fed and passaged as described in the text without centrifugation in 96-well plates for 22 or 23 passages then fixed and stained for pluripotency markers Oct4, Nanog, or Ssea4 with DAPI counterstain. White bar equals $100 \mu \mathrm{m}$. (M-N) Parallel cultures to those described in A were submitted for karyotype analysis which revealed a normal complement of 46 chromosomes. Please click here to view a larger version of this figure. 


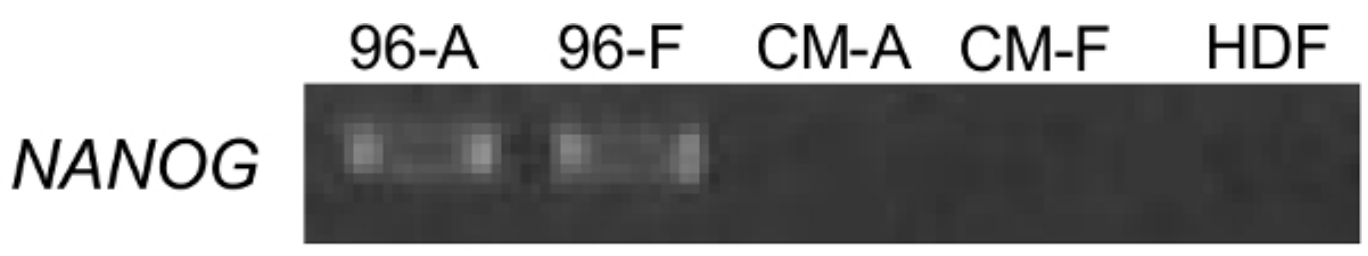

\section{POU5F1}

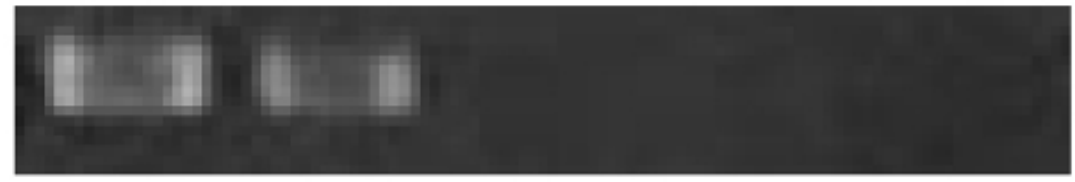

REX1

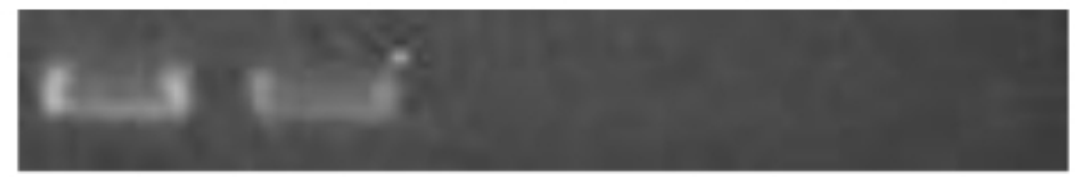

MYL7

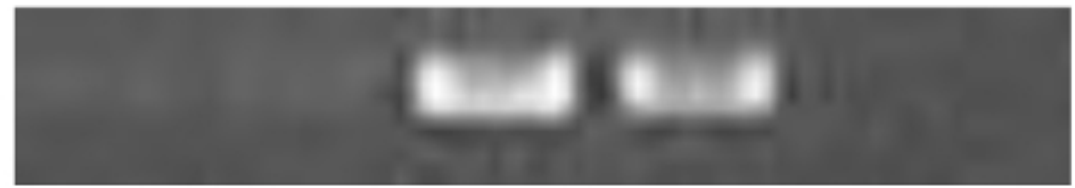

\section{GAPDH}

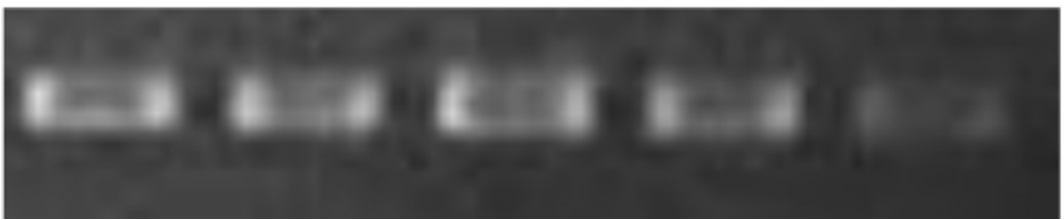

Figure 6. Adipose (96-A) and fibroblast (96-F) derived iPSCs cultured for more than 20 passages in the 96-well robotic format maintained pluripotency gene expression and differentiated into cardiomyocytes. Total mRNA was extracted from both robotically cultured iPSC lines as well as cardiomyocytes differentiated from both lines (CM-A=adipose iPSC derived cardiomyocytes, CM-F=fibroblast iPSC derived cardiomyocytes, collected 14 days after differentiation induction) and used for RT-PCR to probe pluripotency markers NANOG, POU5F1, REX1, cardiomyocyte marker MYL7 and loading control GAPDH. Human dermal fibroblasts (HDF) were also collected and used as a non-iPSC, noncardiomyocyte control. Please click here to view a larger version of this figure.

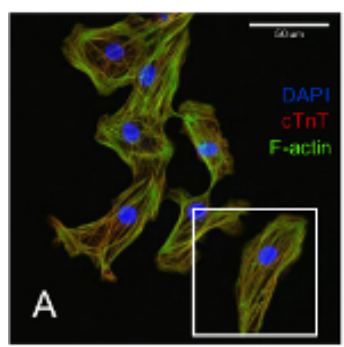

Fibroblast iPSC derived cardiomyocytes
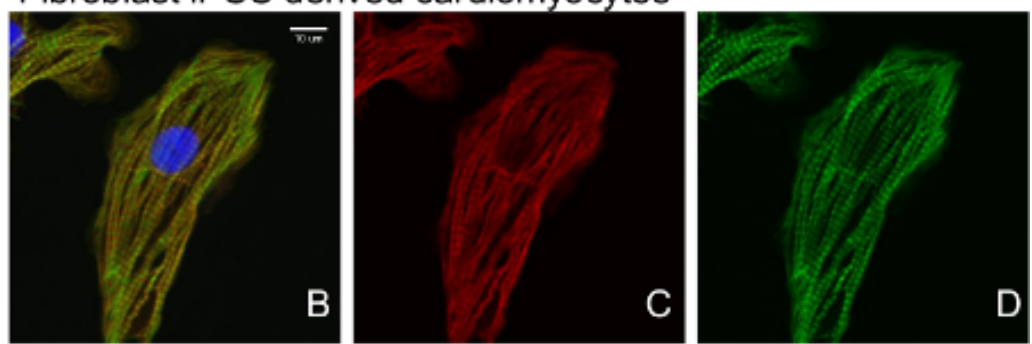

\section{Adipose iPSC derived cardiomyocytes}
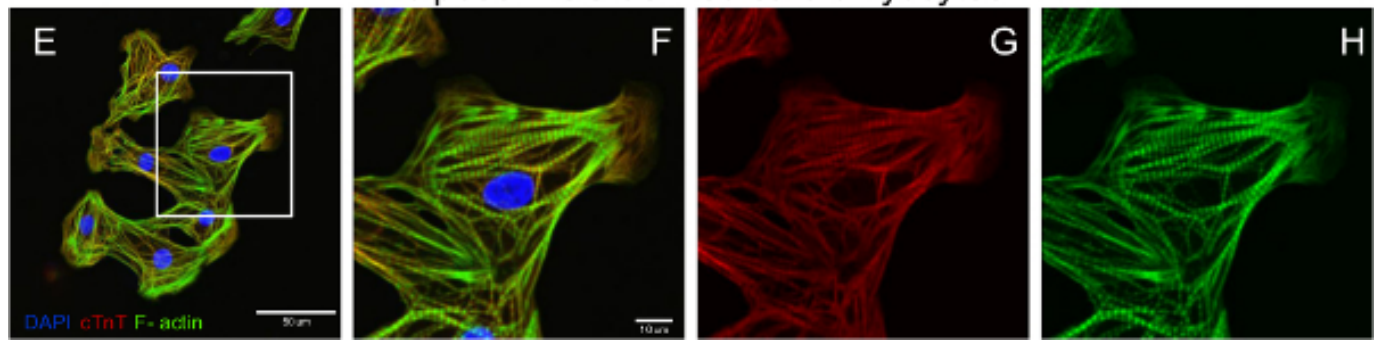

Figure 7. Fibroblast and adipose derived iPSCs maintained the ability to differentiate into cardiomyocytes after long term 96 -well robotic culture. (A-H) Adipose and fibroblast iPSCs cultured in the 96-well robotic format for more than 20 passages were differentiated into cardiomyocytes. 14 days after differentiation induction, 96-well plate bound cardiomyocytes were dissociated and replated at a low density onto glass slides for immunostaining of troponin T (red), F-actin (green) and nuclei (blue). Please click here to view a larger version of this figure. 


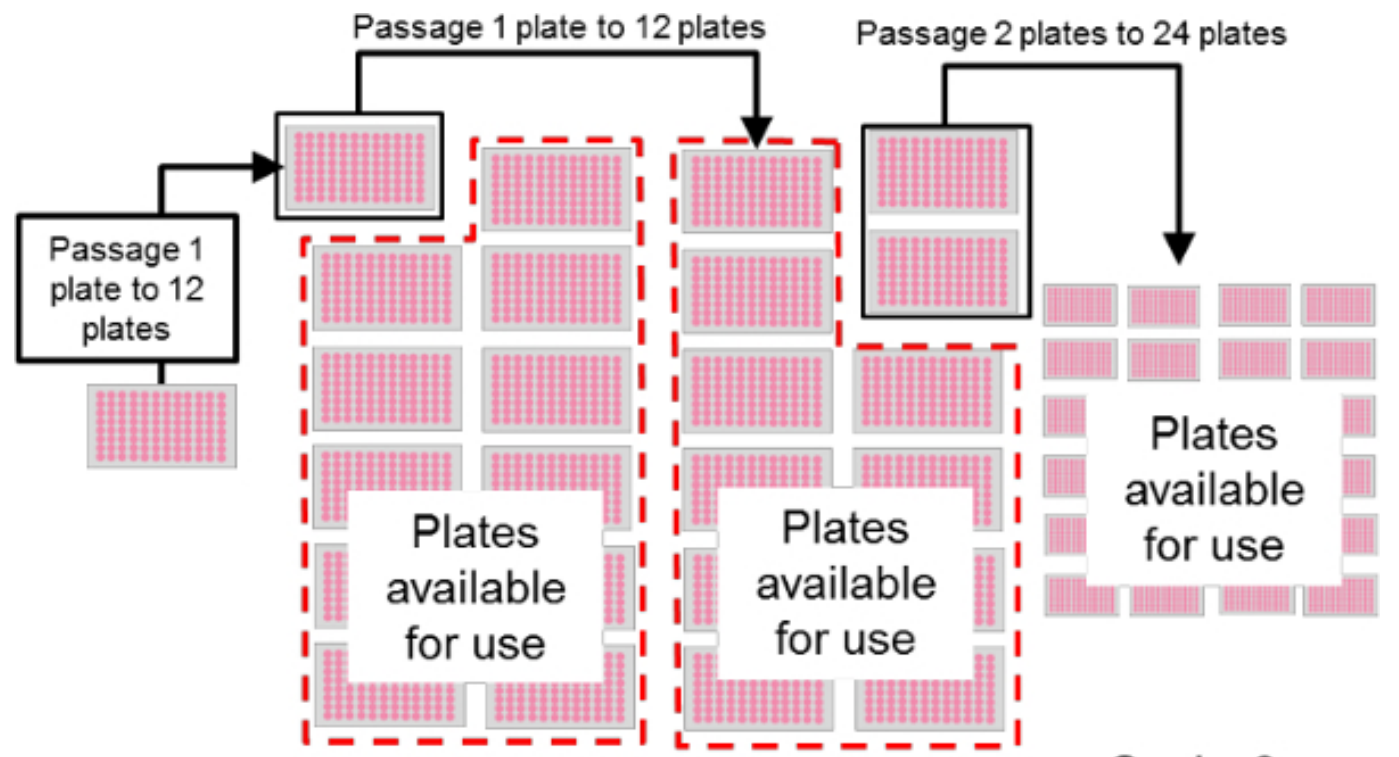

\section{Cycle 0}

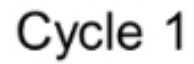

Cycle 2

Cycle 3

Figure 8. Scaling up 96-well plate stem cell culture. Scale up of stem cell production depends on the number of plates used to seed the subsequent group of plates. The user can either maintain one production level by passaging the same number of plates at each split opportunity or expand production by seeding more plates. For example, in Cycle 0, one 96-well plate is passaged to twelve new plates (Cycle 1), creating a 12 fold expansion. This rate may be maintained if one of the twelve plates is passaged to a new set of twelve plates (Cycle 1 to 2 ) or expanded by passaging multiple plates (Cycle 2 to 3 ). During passage, any plates not used to maintain the colony are available for downstream applications. Therefore, passaging 12 plates routinely could yield as many as 144 plates in two passages: 12 for colony maintenance and 132 for other uses. Please click here to view a larger version of this figure. 


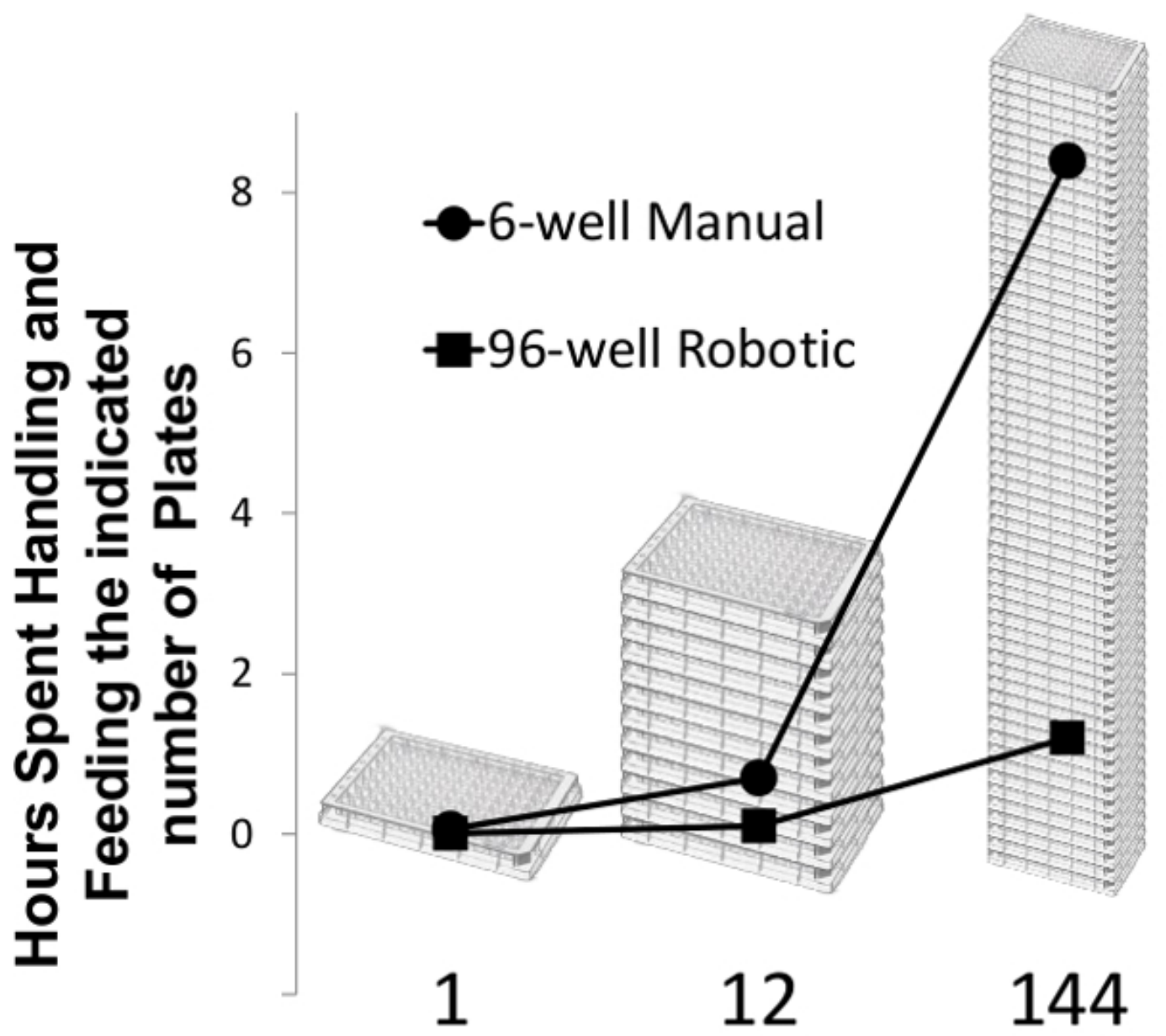

\section{The number of plates handled/fed}

Figure 9. Efficiency of 96-well plate based culture for cell production greatly exceeds manual cultivation. As noted in Figure 8 , one plate can be passaged to seed twelve plates, which can then be passaged to 144 plates. This rate of expansion can be achieved in two split opportunities. A technician requires about 3.5 minutes to feed one 6-well plate whereas they spend about 30 sec with one 96 -well plate to examine it on the microscope and place it on the robot. If the technician is carrying 144 plates, they will spend about $1.2 \mathrm{hr}$ handling plates when feeding with the robot whereas manual culture will require a dedicated $8 \mathrm{hr}$ to feed. Please click here to view a larger version of this figure.

\section{Discussion}

In the present study we developed a method of robotic culture for iPSC production that miniaturizes and automates feeding and passage in a 96-well plate format while also enabling efficient scale up. A liquid handling robot was used to routinely feed iPSC colonies and passage them at regular intervals by enzymatic dissociation. The robot was also programmed to produce extracellular matrix gel coated plates and perform a seeding density gradient. When fibroblast and adipose derived iPSCs were cultured in this system for greater than 20 passages, about 3 months, pluripotency was maintained, as were stable karyotypes. Both cell lines were capable of differentiation into cardiomyocytes. Together, the collection of protocols described here enables users with very little stem cell culture experience, or limited access to specialized culture equipment, to culture stem cells and achieve high cell production or multi-line handling with minimal labor and cost.

The robotic protocols detailed here provide a method for economical scale up of iPSC production and handling. One advantage is scalable stem cell culture in an established plate based format demonstrated here and previously ${ }^{44,47-49}$ to maintain pluripotency. While other formats of scalable stem cell culture yield pluripotent cells $\mathrm{s}^{20,23,29}$ this plate based method requires essentially no change in culture format, like adaption to suspension, use of antifoaming chemicals or prolonged use of Rho-kinase inhibitor ${ }^{20}$. This plate based method can therefore rapidly, and predictably, accommodate new lines because the culture conditions are similar. As the use of iPSCs for disease modeling increases ${ }^{4,6,19,50}$, new iPSC lines are continuously generated. The techniques described here are best suited to culture new lines in medium to high volume and throughput because the barrier to transition onto the format is low. This is also true for the labor and equipment necessary to maintain the colonies. Finally, because the format and handling are similar to the environment used to derive and validate iPSC lines, culture performance, such as directed differentiation, is likely to be more predictable.

Figure 8 illustrates one strategy for scale up of stem cell production in 96-well plates. One plate (Cycle 0 ) is used to robotically seed 12 new plates, a 12 fold increase (Cycle 0 to 1 ). After several days of feeding, one of the twelve plates is passaged to seed another set of twelve plates 
(Cycle 1 to Cycle 2). The remaining eleven plates are now available for other uses and represent the production component of the system. At this rate, passage of one plate will provide 11 plates every cycle, or every 3-4 days. This methodology can be scaled more when multiple plates are passaged as shown in the transition from Cycle 2 to Cycle 3. In Cycle 3, two plates are always used to maintain the colony and seed 24 new plates. The remaining 22 plates are then available for use after every passage cycle. At any point in the maintenance cycle the user can seed more plates to increase production. One example would be to passage every column for two growth cycles. The first passage converts one plate into twelve plates, and each of the twelve plates is used to seed 144 plates. In this case, a user starts with one plate and after two passages, or about 1.5-2 weeks of culture, has 144 plates. For example, the fibroblast and adipose iPSC lines yield approximately 25-75 million cells per 96well plate when ready to passage. 144 plates could therefore represent about $4-7 \times 10^{9}$ cells.

What is the labor burden for carrying 144 96-well plates robotically? One of the goals for this work was scalable stem cell production that reduced labor compared to traditional (i.e., 6-well plate) stem cell culture. The robot accomplishes this by alleviating the need to physically handle each plate during feeding and passaging. While 96-well plate production scales linearly like it would in traditional 6-well plates, the time a technician spends working with the plates is significantly less using robotic culture. The production efficiency of robotic culture is derived from this difference (Figure 9). It was found technicians could remove a 6-well plate from the incubator, check it on the microscope, then aspirate and feed the plate in approximately 3.5 minutes. In comparison, technicians spend approximately 30 sec removing a 96-well plate from the incubator and inspecting it on the microscope for density and contamination before placing it on the robot. With an expanded feeding protocol similar to the one described above, six 96-well plates can be loaded and fed, which takes approximately 21 min, or 3.5 min per plate. The total elapsed time to feed a plate is comparable between robotic and manual methods, but to hand feed 6 plates a technician is required for all 21 min whereas the technician spends only $3 \mathrm{~min}$ handling the six plates for the robot (a $30 \mathrm{sec}$ inspection per plate). As Figure 9 shows, the time a technician spends handling 144 plates using the robot is about $1.2 \mathrm{hr}$ as opposed to $8 \mathrm{hr}$ manually and the robot will never make a mistake handling the plates or transferring liquids.

The 96-well iPSC culture methods described here provide a tractable platform for complex screening tasks. Because each well is genetically identical and seeded at the same density from the same initial pool, variables can be distributed across the plate and maintained by the robot with commercially available reservoirs to segregate conditions. This would be useful for experiments such as stem cell growth media development ${ }^{34,35,47}$, substrate or culture condition testing and toxicity studies utilizing stem cells ${ }^{51}$. Since each stem cell line is unique in terms of optimal colony size and passage requirements, one can use this system to modulate seeding density, feeding frequency and passage handling by manipulating the columns harvested, the mechanical agitation during passage and the feeding frequency. Iterating through these variables will allow the user to quickly find a set of conditions suitable for culture of a new line or develop new culture techniques. The robotic system is also capable of maintaining 96 parallel but independent stem cell colonies. When iPSC are derived from somatic cells or cloned after genetic manipulation, many parallel clones are screened to yield potential iPSC lines. Once single cells are seeded into 96-well plates, this system can feed and passage the 96-well plates keeping each colony segregated. This enables very high throughput when selecting potential clones and eliminates the difficulty in physically culturing 96 parallel lines. This method also allows scaled up production of each clone so that material is readily available for parallel analysis. Finally, we envision integrating these culture techniques with a robotic plate trafficking system that delivers plates to and from the incubator enabling fully automated culture. This could be accomplished with more complex robotics that allow for greater expansion since the basic protocols described here are transferable to other plate based systems.

The first critical steps to address in the protocols are ones that prevent and check for contamination such as steps 1.5 and 4.2 . Contamination, as discussed above, can be successfully avoided if good sterile technique and common sense are utilized. It is imperative, regardless of stem cell culture format, that the operator check for contamination and doing so in this protocol at the indicated steps will significantly reduce the risk for contamination. Proper extracellular matrix gel application is a second essential step to the overall success of this protocol. Without properly coating the 96-well plates, the stem cells will not grow. One common issue is incomplete well coating. Experience has shown that air bubbles, electrostatic attraction and capillary action hinder complete well coating. The pre-wetting step (1.6) was developed to significantly improve well bottom coating and should not be skipped. This pre-wetting step appears to reduce the apparent hydrophobicity of the plastic 96 -well plate such that when the extracellular matrix gel coating is applied it is evenly distributed across the well bottom and sides. It is also recommended to gently tap the 96-well plates against a clean hand once coated to ensure even extracellular matrix gel solution distribution. The dissociation parameters are also important to optimize. Extended incubation with enzyme or EDTA based dissociation reagent will result in single cells which may or may not be the goal during passage. Therefore, the operator should pay special attention to how dissociation time, trituration force, and wash repetitions affect colony morphology and health and adjust accordingly.

Understanding the limitations to the techniques described here are paramount to successful operation. As in any other cell culture setting, the use of 96-well plates presents a relatively high contamination risk. As described above, a technician is handling each plate during feeding and passaging; for example, when opening the lid, putting the plate on the robot bed, and checking the plate on the microscope. Therefore, as noted after step 1.5, it is imperative not to touch the inside of any plate lid or expose this part to any potentially contaminated surface such as the sloped heating blocks or vertical pins on the bed. During protocol development this limitation was discovered and was the impetus to develop a rack to hold plate lids to maintain sterility. Likewise, the trough reservoirs, pipet tips and other supplies on the liquid handling robot bed are potential sources of contamination because they are open to environment and handled by the technician. Therefore, good sterile technique should be applied such as reducing movements over exposed culture materials or open liquids. Another limitation is that when the protocol is scaled up, visually checking every well of $>100$ 96-well plates is cumbersome. This can be dealt with by visually inspecting the whole plate for signs of contamination, such as turbid media, to identify suspicious wells. For future scale up, use of an automated microscope and indicator of cell growth and potential contamination will be incorporated.

In summary, the high-throughput 96-well based platform described here offers a reproducible, high fidelity method for stem cell culture and production in a plate format. This method reduces the requisite experience, equipment necessary and labor time dedicated to stem cell culture while maintaining the benefits of traditional adherent culture. 


\section{Disclosures}

The authors MKC, MJG, EEB, NJD and RO are or were at the time of development employees of InvivoSciences, INC which conceived of and developed the robotic protocols described here. TW is the CSO for InvivoSciences INC. SH is an employee of Gilson INC.

\section{Acknowledgements}

This work has been supported in part by NIH grants, R44 GM087784 and R01 HL109505. Authors thank the technical and OEM team at Gilson, INC for extended technical support.

\section{References}

1. Takahashi, K., Okita, K., Nakagawa, M., Yamanaka, S. Induction of pluripotent stem cells from fibroblast cultures. Nature protocols. 2, (12), 3081-3089 (2007).

2. Grskovic, M., Javaherian, A., Strulovici, B., Daley, G. Q. Induced pluripotent stem cells--opportunities for disease modelling and drug discovery. Nature reviews. Drug discovery. 10, 915-929 (2011).

3. Shi, Y. Induced pluripotent stem cells, new tools for drug discovery and new hope for stem cell therapies. Current Molecular Pharmacology. 2, $15-18(2009)$

4. Xu, X., Zhong, Z. Disease modeling and drug screening for neurological diseases using human induced pluripotent stem cells. Nature Publishing Group. 34, (6), 755-764 (2013).

5. Kirouac, D. C., Zandstra, P. W. The systematic production of cells for cell therapies. Cell stem cell. 3, (4), 369-381 (2008).

6. Ebert, P., Liang, J. W. Induced Pluripotent Stem Cells as a Disease Modeling and Drug Screening Platform. Journal of Cardiovascular Pharmacology. 60, (4), 408-416 (2012).

7. Pa Lalit,, Hei, D. J., Raval, A. N., Kamp, T. J. Induced pluripotent stem cells for post-myocardial infarction repair: remarkable opportunities and challenges. Circulation research. 114, 1328-1345 (2014).

8. Compagnucci, C., Nizzardo, M., Corti, S., Zanni, G., Bertini, E. In vitro neurogenesis: development and functional implications of iPSC technology. Cellular and Molecular Life Sciences. 1-17 (2013).

9. Yi, F., Liu, G. -H., Belmonte, J. C. I. Human induced pluripotent stem cells derived hepatocytes: rising promise for disease modeling, drug development and cell therapy. Protein \& Cell. 3, 246-250 (2012).

10. Mummery, C. L., Zhang, J., Ng, E. S., Elliott, D. a, Elefanty, aG., Kamp, T. J. Differentiation of Human Embryonic Stem Cells and Induced Pluripotent Stem Cells to Cardiomyocytes: A Methods Overview. Circulation Research. 111, (3), 344-358 (2012).

11. Okano, H., et al. Steps toward safe cell therapy using induced pluripotent stem cells. Circulation Research. 112, 523-533 (2013).

12. Scheiner, Z. S., Talib, S., Feigal, E. G. The Potential for Immunogenicity of Autologous Induced Pluripotent Stem Cell-derived Therapies. The Journal of biological chemistry. 289, 4571-4577 (2014).

13. Sun, N., et al. Patient-specific induced pluripotent stem cells as a model for familial dilated cardiomyopathy. Science translational medicine. 4 , (130), 130ra47 (2012).

14. Gunaseeli, I., Doss, M. X., Antzelevitch, C., Hescheler, J., Sachinidis, A. Induced pluripotent stem cells as a model for accelerated patientand disease-specific drug discovery. Current medicinal chemistry. 17, 759-766 (2010).

15. Zweigerdt, R. Large scale production of stem cells and their derivatives. Advances in Biochemical Engineering/Biotechnology. 114, 201-235 (2009).

16. Serra, M., Brito, C., Correia, C., Alves, P. M. Process engineering of human pluripotent stem cells for clinical application. Trends in Biotechnology. 30, 350-359 (2012).

17. Lock, L. T., Tzanakakis, E. S. Stem/Progenitor cell sources of insulin-producing cells for the treatment of diabetes. Tissue engineering. 13, 1399-1412 (2007).

18. Jing, D., Parikh, A., Canty, J. M., Tzanakakis, E. S. Stem cells for heart cell therapies. Tissue engineering. Part B, Reviews. 14, 393-406 (2008).

19. Soldner, F., Jaenisch, R. iPSC Disease Modeling. Science. 338, 1155-1156 (2012).

20. Olmer, R., et al. Long term expansion of undifferentiated human iPS and ES cells in suspension culture using a defined medium. Stem cell research. 5, (1), 51-64 (2010).

21. Wang, Y., Chou, B. -K., Dowey, S., He, C., Gerecht, S., Cheng, L. Scalable expansion of human induced pluripotent stem cells in the defined xeno-free E8 medium under adherent and suspension culture conditions. Stem cell research. 11, (3), 1103-1116 (2013).

22. Chen, V. C., et al. Scalable GMP compliant suspension culture system for human ES cells. Stem cell research. 8, (3), 388-402 (2012).

23. Amit, M., Laevsky, I., Miropolsky, Y., Shariki, K., Peri, M., Itskovitz-Eldor, J. Dynamic suspension culture for scalable expansion of undifferentiated human pluripotent stem cells. Nature protocols. 6, (5), 572-579 (2011).

24. Olmer, R., et al. Suspension Culture of Human Pluripotent Stem Cells in Controlled. Stirred Bioreactors. Tissue engineering. Part C. 18, (10), (2012).

25. Kehoe, D. E., Jing, D., Lock, L. T., Tzanakakis, E. S., Ph, D. Scalable Stirred-Suspension Bioreactor Culture. Tissue engineering. Part A. 16, (2), (2010).

26. Oh, S. K. W., et al. Long-term microcarrier suspension cultures of human embryonic stem cells. Stem Cell Research. 2, 219-230 (2009).

27. Chen, A. K. -L., Reuveny, S., Oh, S. K. W. Application of human mesenchymal and pluripotent stem cell microcarrier cultures in cellular therapy: achievements and future direction. Biotechnology advances. 31, 1032-1046 (2013).

28. Yang, H. S., Jeon, O., Bhang, S. H., Lee, S. -H., Kim, B. -S. Suspension culture of mammalian cells using thermosensitive microcarrier that allows cell detachment without proteolytic enzyme treatment. Cell transplantation. 19, 1123-1132 (2010).

29. Zweigerdt, R., Olmer, R., Singh, H., Haverich, A., Martin, U. Scalable expansion of human pluripotent stem cells in suspension culture. Nature. 6, (5), (2011). 
30. Kami, D., et al. Large-scale cell production of stem cells for clinical application using the automated cell processing machine. BMC biotechnology. 13, 102 (2013).

31. Hussain, W., Moens, N., Veraitch, F. S., Hernandez, D., Mason, C., Lye, G. J. Reproducible culture and differentiation of mouse embryonic stem cells using an automated microwell platform. Biochemical engineering journal. 77, (100), 246-257 (2013).

32. Tandon, N., Marolt, D., Cimetta, E., Vunjak-Novakovic, G. Bioreactor engineering of stem cell environments. Biotechnology Advances. 31, 1020-1031 (2013).

33. Sun, N., et al. Feeder-free derivation of induced pluripotent stem cells from adult human adipose stem cells. Proceedings of the National Academy of Sciences of the United States of America. 106, 15720-15725 (2009).

34. Ludwig, T. E., Bergendahl, V., Levenstein, M. E., Yu, J., Probasco, M. D., Thomson, J. A. Feeder-independent culture of human embryonic stem cells. Nature. 3, 637-646 (2006).

35. Chen, G., Gulbranson, D. R., et al. Chemically defined conditions for human iPSC derivation and culture. Nature methods. 8, (5), 424-429 (2011).

36. Kinney, M. A., Sargent, C. Y., McDevitt, T. C. The multiparametric effects of hydrodynamic environments on stem cell culture. Tissue engineering. Part B, Reviews. 17, 249-262 (2011).

37. Villa-Diaz, L. G., Ross, A. M., Lahann, J., Krebsbach, P. H. Concise review: The evolution of human pluripotent stem cell culture: from feeder cells to synthetic coatings. Stem cells (Dayton, Ohio). 31, (2013).

38. Park, I. H., et al. Reprogramming of human somatic cells to pluripotency with defined factors. Nature. 451, (7175), 141-146 (2008).

39. Chambers, I., et al. Functional expression cloning of Nanog, a pluripotency sustaining factor in embryonic stem cells. Cell. 113, (5), (2003).

40. Wright, A. J., Andrews, P. W. Surface marker antigens in the characterization of human embryonic stem cells. Stem cell research. 3, (1), 3-11 (2009).

41. Thomson, J. a Embryonic Stem Cell Lines Derived from Human Blastocysts. Science. 282, (5391), 1145-1147 (1998).

42. Mayshar, Y., et al. Identification and classification of chromosomal aberrations in human induced pluripotent stem cells. Cell stem cell. 7, (4), 521-531 (2010).

43. Martins-Taylor, K., Xu, R. -H. Concise review: Genomic stability of human induced pluripotent stem cells. Stem cells(Dayton, Ohio). 30, (1), 22-27 (2012)

44. Okita, K., Ichisaka, T., Yamanaka, S. Generation of germline-competent induced pluripotent stem cells. Nature. 448, (7151), 313-317 (2007).

45. Aasen, T., et al. Efficient and rapid generation of induced pluripotent stem cells from human keratinocytes. Nature. 26, (11), 1276-1284 (2008).

46. Lian, X., et al. Robust cardiomyocyte differentiation from human pluripotent stem cells via temporal modulation of canonical Wnt signaling. Proceedings of the National Academy of Sciences of the United States of America. 109, (27), E1848-E1857 (2012).

47. Ludwig, T. E., et al. Derivation of human embryonic stem cells in defined conditions. Nature biotechnology. 24, (2), 185-187 (2006).

48. Wang, L., Li, L., Menendez, P., Cerdan, C., Bhatia, M. Human embryonic stem cells maintained in the absence of mouse embryonic fibroblasts or conditioned media are capable of hematopoietic development. Blood. 105, (12), 4598-4603 (2005).

49. Yu, J., et al. Induced pluripotent stem cell lines derived from human somatic cells. Science(New York, N. Y.). 318, (5858), 1917-1920 (2007).

50. Kim, C. Disease modeling and cell based therapy with iPSC: future therapeutic option with fast and safe application. Blood research. 49, 7-14 (2014).

51. Jung, E. -M., et al. Evaluation of developmental toxicity using undifferentiated human embryonic stem cells. Journal of applied toxicology: JAT. (February. (2014). 